Please do not remove this page

RMIT

UNIVERSITY

\title{
A Probabilistic Tree-Based Representation for Non-convex Minimum Cost Flow Problems
}

Ghasemishabankareh, Behrooz; Ozlen, Melih; Neumann, Frank; Li, Xiaodong

https://researchrepository.rmit.edu.au/esploro/outputs/9921863538201341/filesAndLinks?institution=61RMIT_INST\&index=null

Ghasemishabankareh, B., Ozlen, M., Neumann, F., \& Li, X. (2018). A Probabilistic Tree-Based

Representation for Non-convex Minimum Cost Flow Problems. Proceedings of the 15th International Conference on Parallel Problem Solving from Nature (PPSN'2018), 69-81.

https://doi.org/10.1007/978-3-319-99253-2_6

Document Version: Accepted Manuscript

Published Version: https://doi.org/10.1007/978-3-319-99253-2_6

Repository homepage: https://researchrepository.rmit.edu.au

(c) Springer Nature Switzerland AG 2018

Downloaded On 2023/04/26 18:55:47 +1000 
Thank you for downloading this document from the RMIT Research Repository.

The RMIT Research Repository is an open access database showcasing the research outputs of RMIT University researchers.

RMIT Research Repository: http://researchbank.rmit.edu.au/

\section{Citation:}

Ghasemishabankareh, B, Ozlen, M, Neumann, F and Li, X 2018, 'A probabilistic tree-based representation for non-convex minimum cost flow problems', in Proceedings of the 15th International Conference on Parallel Problem Solving from Nature (PPSN'2018), Coimbra, Portugal, 8 - 12

September 2018, pp. 69-81.

See this record in the RMIT Research Repository at:

https://researchbank.rmit.edu.au/view/rmit:52635

Version: Accepted Manuscript

Copyright Statement:

(C) Springer Nature Switzerland AG 2018

\section{Link to Published Version:}

https://doi.org/10.1007/978-3-319-99253-2_6 


\title{
A Probabilistic Tree-Based Representation for Non-convex Minimum Cost Flow Problems
}

\author{
Behrooz Ghasemishabankareh ${ }^{\star 1}$, Melih Ozlen ${ }^{1}$, Frank Neumann ${ }^{2}$, and \\ Xiaodong $\mathrm{Li}^{1}$ \\ 1 School of Science, RMIT University, Melbourne, Australia \\ \{behrooz.ghasemishabankareh,melih.ozlen, xiaodong.li\}@rmit.edu.au \\ 2 School of Computer Science, The University of Adelaide, Adelaide, Australia \\ frank.neumann@adelaide.edu.au
}

\begin{abstract}
Network flow optimisation has many real-world applications. The minimum cost flow problem (MCFP) is one of the most common network flow problems. Mathematical programming methods often assume the linearity and convexity of the underlying cost function, which is not realistic in many real-world situations. Solving large-sized MCFPs with nonlinear non-convex cost functions poses a much harder problem. In this paper, we propose a new representation scheme for solving non-convex MCFPs using genetic algorithms (GAs). The most common representation scheme for solving the MCFP in the literature using a GA is priority-based encoding, but it has some serious limitations including restricting the search space to a small part of the feasible set. We introduce a probabilistic tree-based representation scheme (PTbR) that is far superior compared to the priority-based encoding. Our extensive experimental investigations show the advantage of our encoding compared to previous methods for a variety of cost functions.
\end{abstract}

Keywords: Representation scheme $\cdot$ genetic algorithm $\cdot$ minimum cost flow problem. mixed integer nonlinear programming.

\section{Introduction}

Network flow problems have numerous applications in electrical and power networks, telecommunication, road and rail networks, and airline services [2]. Different types of network flow problems exist, e.g., the shortest path problem, the maximum flow problem, the assignment problem, the transportation problem, and the minimum cost flow problem (MCFP), among which MCFP is one of the most general cases with applications such as distribution problems, optimal loading of a Hopping aeroplane and the racial balancing of schools [2].

MCFPs can be formulated and solved by Linear Programming (LP) techniques, when the underlying cost function is linear or can be approximated by a linear function [18]. However, many real-world MCFPs are nonlinear and require formulation using a nonlinear cost function, instead of a linear approximation.

\footnotetext{
* Corresponding author.
} 
For example, in a transportation problem, the nonlinearity of a cost function is due to the economy of scale phenomenon, which occurs when cost per unit of the transportation flow decreases with an increasing amount of the total flow [7]. Many studies suggest the appropriateness of employing nonlinear cost functions in the network design problems $[4,16]$.

Some attempts have been made in using genetic algorithms (GAs) to solve the network flow problems $[1,7,14]$. Among these works, the representation scheme plays a critical role in their success. Several representation schemes exist for the network flow problems such as variable-length encoding [20], fixed-length encoding [3], and priority-based representation (PbR) [17]. The most common representation scheme for solving MCFPs is PbR [8]. PbR scheme has been used to solve the shortest path problems, the transportation problems, as well as the network design problems $[8,14,17]$. Although $\mathrm{PbR}$ is widely used for solving network flow problems, it has some serious drawbacks (when dealing with MCFP), most noticeably its restriction on any search algorithm from reaching some parts of the feasible search space (see Section 2 for details).

To counteract the above limitations, in this paper we propose a probabilistic tree-based representation (PTbR) for solving nonlinear non-convex MCFP instances using the GA. The PTbR allows all possible feasible solutions to be generated, instead of being restricted to a small part of the feasible region (e.g., $\mathrm{PbR}$ scheme). This paper first examines the capabilities of PTbR and compare it with that of the PbR scheme. Then a comparative study is carried out on the performance of the GA employing these two different representation schemes on a set of 35 benchmark instances. This paper has the following contributions: 1) proposing a novel representation scheme (PTbR) to deal with MCFP; 2) providing a close examination between PTbR and PbR to find out which one is more effective for handling MCFPs; 3) conducting extensive experiments to compare the performance of the PTbR-based GA (PtGA) variants with the PbR-based GA (PrGA) for solving non-convex MCFP instances. We also compare our results with those of the mathematical solver packages.

The rest of the paper is structured as follows: Section 2 gives the preliminaries and Section 3 describes our proposed probabilistic tree-based representation and the GA employing PTbR scheme for solving MCFPs. The experimental studies are presented in Section 4 and Section 5 provides the conclusion.

\section{Preliminaries}

This section describes the problem definition, the $\mathrm{PbR}$, and finally discusses the drawbacks of $\mathrm{PbR}$. Let $G(N, A)$ be a network consisting of a set $N$ of $n$ nodes and a set $A$ of $m$ directed arcs. The maximum and minimum amount of flow on each arc $(i, j)$ are equal to $u_{i j}$ and 0 , respectively. $b(i)$ denotes the amount of supply or demand for source or sink node. $b(i)>0$ denotes that node $i$ is a supply node and $b(i)<0$ shows that node $i$ is a demand node with a demand of $-b(i)$ and $b(i)=0$ denotes the transshipment node $i$. Fig. 1 shows an example of the MCFP with $n=5$ nodes and $m=7$ arcs, which has one supplier node $(b(1)=10)$ 


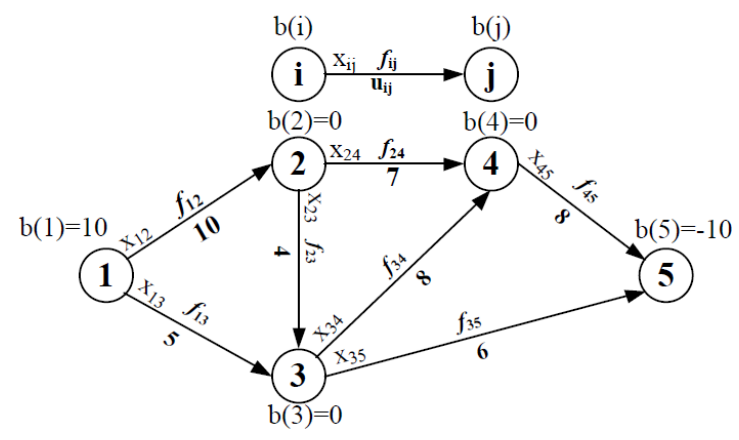

Fig. 1: An example of the MCFP $(n=5, m=7)$.

and one demand node $(b(5)=-10)$. In this example, we aim to satisfy the demand by sending all supplies through the network while minimising the total cost. The integer flow on an arc $(i, j)$ is represented by $x_{i j}$ and the associated cost for the flow $\left(x_{i j}\right)$ is denoted by $f_{i j}\left(x_{i j}\right)$. The formulation of the MCFP is as follows [2]:

$$
\begin{gathered}
\text { Minimise }: z(\mathbf{x})=\sum_{(i, j) \in A} f_{i j}\left(x_{i j}\right), \\
\text { s.t. } \sum_{\{j:(i, j) \in A\}} x_{i j}-\sum_{\{j:(j, i) \in A\}} x_{j i}=b(i) \quad \forall i \in N, \\
0 \leq x_{i j} \leq u_{i j} \quad \forall \quad(i, j) \in A, \\
x_{i j} \in Z \quad \forall \quad(i, j) \in A,
\end{gathered}
$$

where Eq.1 minimises the total cost through the network. Eq. 2 is a flow balance constraint which states the difference between the total outflow (first term) and the total inflow (second term). The flow on each arc should be between an upper bound and zero (Eq. 3), and finally all the flow values are integer numbers (Eq. 4). In this paper we consider the following assumptions for the MCFP: 1) the network is directed; 2) there are no two or more arcs with the same tail and head in the network; 3) the single-source single-sink MCFP is considered; 4) the total demands and supplies in the network are equal, i.e., $\sum_{i=1}^{n} b(i)=0$.

\subsection{Priority-based representation}

Priority-based representation $(\mathrm{PbR})$ is the most commonly-used representation method for MCFPs [8]. In order to represent a candidate solution for an MCFP, $\mathrm{PbR}$ lets the number of genes to be equal to $n$ and the value of each gene is generated randomly between 1 and $n$, which represents the priority of each node for constructing a path among all possible nodes [8]. Fig. 2a illustrates the $\mathrm{PbR}$ chromosome for the network presented in Fig. 1. In order to obtain a feasible solution, a two-phase decoding procedure is followed. In phase I, a path is generated based on the priorities and the maximum possible flow is sent through the generated path in phase II. After sending the flow on the network, the upper 


\begin{tabular}{c|c|c|c|c|c|}
\multicolumn{1}{c}{ Node: } & 1 & 2 & 3 & 4 & 5 \\
\hline Chromosome & 5 & 5 & 2 & 3 & 1 \\
\hline
\end{tabular}

(a) An example of a chromosome for the network in Fig. 1.

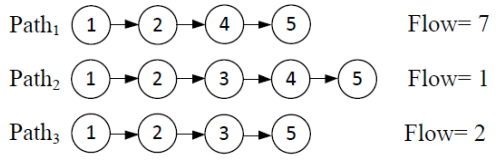

(b) A feasible solution for the given chromosome.

Fig. 2: The PbR chromosome and its corresponding solution.

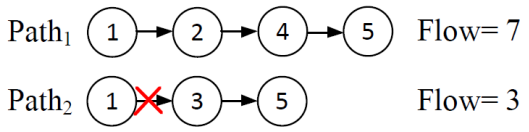

Fig. 3: A feasible solution that PbR fails to represent (for the network in Fig. 1).

bound $\left(u_{i j}\right)$, supply and demand should be updated. If the supply/demand is not equal to 0 , the next path should be generated. The above procedure repeats until all demands are satisfied. Fig. 2b presents a feasible solution for the given chromosome in Fig. 2a.

Although PbR has been commonly used in the network flow problems, it has some limitations in representing the full extent of the feasible space for MCFP. Fig. 3 shows an example (for the network presented in Fig. 1) that PbR is unable to represent. Here the first path is generated as follows: $1 \rightarrow 2 \rightarrow 4 \rightarrow 5$. Since in Path $_{1}$ after node 1 , node 2 is selected, it shows that node 2 has a higher priority than node 3 . Hence, if arc $(1,2)$ is not saturated, $\mathrm{PbR}$ will not allow any flow to be sent through arc (1,3), essentially blocking this possibility completely (Fig. 3, $\mathrm{Path}_{2}$ ). This means that $\mathrm{PbR}$ is unable to represent a potential feasible solution such that the flow would go through arc (1,3)(as shown in Fig. 3). Another limitation for $\mathrm{PbR}$ is that each time a path is generated, we are supposed to send the maximum possible amount on the generated path. These limitations would restrict a search algorithm from reaching the full extent of the feasible space.

\section{Proposed method}

Representation plays a critical role before applying an optimisation algorithm, and this applies to GA too. In this section we first propose a probabilistic treebased representation (PTbR) scheme for solving MCFPs, which alleviates the deficiency of using PbR. Then we describe the GA employing PTbR for solving MCFP instances.

\subsection{Probabilistic tree-based representation}

To counteract the above-mentioned limitations of the $\mathrm{PbR}$, we propose the $\mathrm{PTbR}$ scheme, where a probability tree is adopted to represent a potential MCFP 


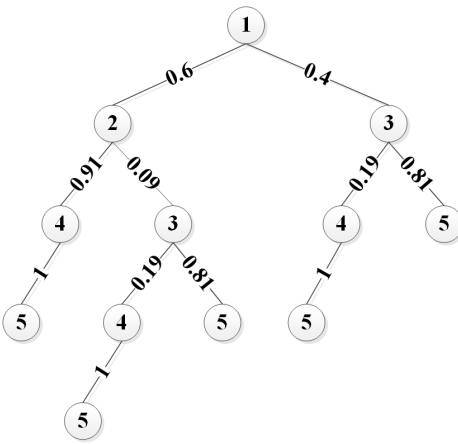

(a) A probability tree structure.

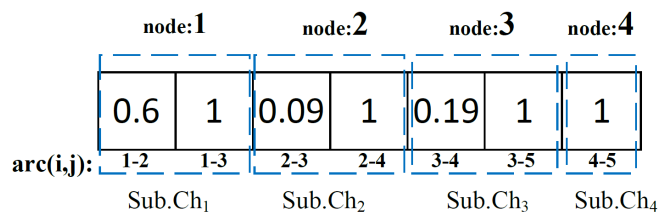

(b) The PTbR based on the probability tree.

Fig. 4: Probability tree and its corresponding PTbR for the network in Fig. 1.

solution. Unlike the PbR scheme which is restricted to a small part of the feasible space, the PTbR is able to represent all possible feasible solutions. Fig.4a shows an example of the probability tree for the network presented in Fig. 1. Here, the probability of each successor node to be selected is defined on each branch.

The tree structure can be converted to a chromosome with several subchromosomes. Fig. 4b shows the PTbR chromosome converted from the probability tree presented in Fig $4 \mathrm{a}$. The PTbR chromosome has $n-1$ sub-chromosomes (Sub.Ch) and the value of each gene is a random number between 0 and 1 which is then accumulated to 1 in each sub-chromosome. In order to obtain a feasible solution from PTbR, in phase I, a path is first constructed, and then a feasible flow is sent through the constructed path in phase II. For example, to obtain a feasible solution for the chromosome in Fig. 4b, we generate the first path from node $i=1\left(\mathrm{Sub}_{\mathrm{C}} \mathrm{Ch}_{i=1}\right)$. A random number is generated in $[0,1]$ ( rand $=0.2)$, and since $0 \leq$ rand $=0.2 \leq 0.6$, we move through arc $(1,2)$ and node 2 is selected. From node $2\left(\mathrm{Sub} \mathrm{Ch}_{i=2}\right)$ another random number is generated $(0.09 \leq$ rand $=0.85 \leq 1)$ and the selected successor node is 4 . From node 4 the only available node is 5 . Hence, the following path is generated: $1 \rightarrow 2 \rightarrow 4 \rightarrow 5$.

In Phase II, we attempt to send a feasible flow through the generated path. First the capacity of the generated path is defined $\left(U=\min \left\{u_{12}=10, u_{24}=\right.\right.$ $\left.\left.7, u_{45}=8\right\}=7\right)$. Then, there are three possible approaches to send a feasible flow on the generated path: 1$)$ send a random flow between 1 and $U(\operatorname{random}(\mathbf{R})) ; 2)$ send a flow 1-by-1 (one-by-one $(\mathbf{O})$ ); 3 ) send the maximum possible amount of the flow on the generated path (maximum $(\mathbf{M})$ ), which is the same as PbR. In the above example, we follow the first approach $(\operatorname{random}(\mathbf{R}))$ and after calculating $U=7$, we send a random flow in $[1,7]$ (e.g., flow $=6$ ) and the network, supply and demand are updated. Since the demand has not been fully met (i.e., not equal to 0 yet), the above procedure is repeated.

Fig. 5 shows a feasible solution for the chromosome presented in Fig. $4 \mathrm{~b}$. Note that in Fig. 5, after generating Path $_{1}$, although arc $(1,2)$ is not saturated, the second path picks node 3 as the successor of node 1 , unlike the PbR. This 


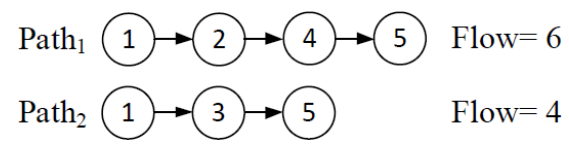

Fig. 5: A feasible solution generated based on the PTbR chromosome in Fig. 4b.

example illustrates that PTbR allows all potential solutions to be generated probabilistically, instead of being restricted by using $\mathrm{PbR}$.

\subsection{Genetic algorithm with PTbR}

This section describes the GA employing the new representation scheme PTbR for solving MCFPs, i.e., PtGA. The key distinction between the PtGA and the PbR-based GA (PrGA) is that PrGA employs the PbR [8]. This PtGA can be described by the following procedure:

Initialisation: First a population with pop_size individuals (chromosomes) is randomly generated. The process of creating a chromosome based on the PTbR is explained in Subsection 3.1.

Crossover and mutation: In order to explore the feasible region, crossover and mutation operators are applied to create the new offspring at each generation. For PtGA, a two-point crossover operation is applied, where two blocks (sub-chromosomes) of the selected chromosome (parents) are first randomly selected. Then, two parents swapping the selected sub-chromosomes to generate new offspring. To perform mutation for PtGA, first a random parent is selected and the randomly chosen sub-chromosome is regenerated to create a new offspring.

Fitness evaluation and selection: For each chromosome in the population, after finding a feasible solution ( $\mathbf{x}$ ) by applying the decoding procedure for PTbR, the value of cost function is evaluated using the following equation: Minimize : $z(\mathbf{x})=\sum_{i=1}^{n} \sum_{j=1}^{n} f\left(x_{i j}\right)$. After calculating the fitness values for all individuals in the population, the tournament selection procedure is applied to select individuals for the next generation.

Termination criteria: The termination criteria for the PtGA are as follows: 1) no further fitness value improvement in the best individual of the population for $\beta$ successive iterations; 2) the maximum number of function evaluations (NFEs) reached. If any of the above conditions is satisfied first, the algorithm stops and the best solution $\left(\mathbf{x}^{*}\right)$ and its corresponding cost function value are reported.

Note that for PrGA, it is common to employ a weight mapping crossover (WMX) and inversion mutation $[12,8]$. The termination criteria can be the same for both PrGA and PtGA.

\section{Experimental Studies}

This section first describes the MCFP instances and cost functions that have been adopted, followed by some discussion about the mathematical solver pack- 


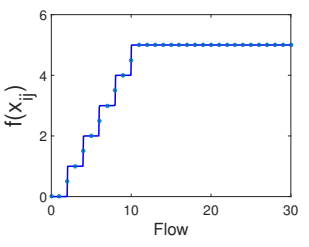

(a) Cost function $F_{1}$

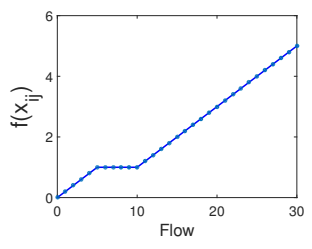

(b) Cost function $F_{2}$

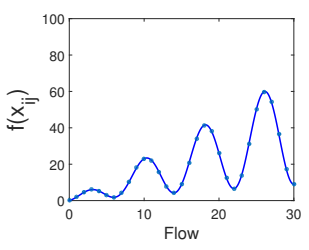

(c) Cost function $F_{3}$

Fig. 6: Shapes of different cost functions.

ages used in our experiments. We then describe the parameter settings, experimental comparisons and result analysis on the performances of PrGA, PtGA, and mathematical solvers in solving these MCFP instances.

Since our focus is to solve nonlinear non-convex MCFP, we adopt a set of nonlinear non-convex cost functions which are commonly-used in the literature $[9,10,15]$. Michalewicz et al. [15] categorised the nonlinear cost functions as 1) piece-wise linear cost functions; 2) multimodal (nonlinear non-convex) cost functions; 3) smooth cost functions which are mostly used for Operations Research (OR) problems. In this paper we chose the nonlinear non-convex and arc-tangent approximation of the piece-wise linear cost functions from $[15,9,10]$ to evaluate the performances of PrGA and PtGA. The formulation of these functions are as follows $[15,9,10]$ :

$$
\begin{gathered}
F_{1}: f\left(x_{i j}\right)=c_{i j}\left(\arctan \left(P_{A}\left(x_{i j}-S\right)\right) / \pi+0.5+\arctan \left(P_{A}\left(x_{i j}-2 S\right)\right) / \pi+0.5+\right. \\
\arctan \left(P_{A}\left(x_{i j}-3 S\right)\right) / \pi+0.5+\arctan \left(P_{A}\left(x_{i j}-4 S\right)\right) / \pi+0.5+ \\
\left.\arctan \left(P_{A}\left(x_{i j}-5 S\right)\right) / \pi+0.5\right) . \\
F_{2}: f\left(x_{i j}\right)=c_{i j}\left(\left(x_{i j} / S\right)\left(\arctan \left(P_{B} x_{i j}\right) / \pi+0.5\right)+\left(1-x_{i j} / S\right)\left(\arctan \left(P_{B}\left(x_{i j}-S\right)\right) / \pi+0.5\right)+\right. \\
\left.\left(x_{i j} / S-2\right)\left(\arctan \left(P_{B}\left(x_{i j}-2 S\right)\right) / \pi+0.5\right)\right) . \\
F_{3}: f\left(x_{i j}\right)=100 \times c_{i j}\left(x_{i j}\left(\sin \left(\frac{5 \pi x_{i j}}{4 S}\right)+1.3\right)\right) .
\end{gathered}
$$

Note that $c_{i j}$ is non-negative coefficient, $P_{A}$ and $P_{B}$ are set to 1000 and $S$ is set to 2 for $F_{1}$, and 5 for $F_{2}$ and $F_{3}$, respectively [10]. All cost functions $F_{1}, F_{2}$ and $F_{3}$ are illustrated in Fig. 6 . A set of 35 single-source single-sink MCFP instances is randomly generated with different number of nodes $(n=$ $\{5,10,20,40,80,120,160\})$ and presented in Table 1 (No. denotes the instance number, and each instance has $n$ nodes and $m$ arcs). Note that, for each node size $(n)$, five different networks are randomly generated. The number of supply/demand for nodes $1 / n$ are set to $q=20 /-20$ in the test instances up to 20 nodes and for all other test problems supply/demand are set to $q=30 /-30$.

This paper focuses on solving nonlinear non-convex MCFPs, which could be considered as mixed integer nonlinear programming (MINLP) problems. However, only very few mathematical solver packages exist for solving MINLP problems, such as CPLEX, Couennn, Baron, LINDOGlobal and AlphaECP [5, 13, 19]. Some of these solvers have serious limitations. For instance, CPLEX is only capable of solving quadratic optimisation problems, BARON cannot handle the 
Table 1: A set of 35 randomly generated single-source single-sink MCFP instances.

\begin{tabular}{|c|c|c|c|c|c|c|c|c|c|c|c|c|c|c|c|c|c|c|c|}
\hline & & $m \mid N$ & $n$ & $m$ & No. & $n$ & $m$ & $N o$ & $n$ & $m$ & No. & $n$ & & $N o$ & $n$ & & No. & $n$ & \\
\hline & & 86 & & 24 & & & 114 & 16 & & 369 & 21 & & 1484 & 26 & & 3419 & 31 & & 4882 \\
\hline & & \begin{tabular}{l|l}
8 & 7
\end{tabular} & & 34 & 12 & & 98 & 17 & & & & & & & & & 32 & & \\
\hline & 5 & $\begin{array}{l}8 \\
8\end{array}$ & 10 & 32 & 13 & 20 & D 105 & 18 & 40 & 37 & 23 & 80 & & & 120 & 3326 & 33 & 160 & \\
\hline & & 99 & & 27 & 14 & & 99 & 1 & & 40 & 24 & & 13 & & & 3212 & 4 & & 4835 \\
\hline & & \begin{tabular}{l|l}
8 & 10
\end{tabular} & & 29 & 15 & & 101 & 20 & & 406 & 25 & & 1526 & 30 & & 2911 & 135 & & 5130 \\
\hline
\end{tabular}

trigonometric functions $\sin (x), \cos (x)$, while Couenne is not able to handle the arctangent function [5]. Among these solvers, AlphaECP and LINDOGlobal are able to handle general MINLPs $[13,19]$. As a result, we choose to compare our PtGA and PrGA results with those of LINDOGlobal and AlphaECP.

\subsection{Parameter settings}

Both PrGA and PtGA are implemented in MATLAB on a PC with Intel(R) Core(TM) i7-6500U $2.50 \mathrm{GHz}$ processor with 8 GB RAM and run 30 times for each problem instance. In order to solve MCFP instances using mathematical solvers, AlphaECP is applied through a high level mathematical language general algebraic modelling system (GAMS) [11] and LINDOGlobal [13] is applied directly on all problem instances.

The parameter settings for the PrGA are as follows: maximum number of iterations $\left(I t_{\max }=200\right)$, population size (pop_size $\left.=\min \{n \times 10,300\}\right)$, crossover rate $\left(P_{c}=0.95\right)$, mutation rate $\left(P_{m}=0.3\right)$ and maximum number of function evaluations $(N F E s=100,000)$. The parameter settings for the PtGA are $I t_{\max }=200$, pop_size $=\min \{n \times 5,300\}, P_{c}=0.95, P_{m}=0.3$ and $N F E s=100,000$. The pop_size value depends on the number of nodes $(n)$ and increases for the larger networks and the $P_{m}=0.3$ value decreases linearly in each iteration. If the results are not improved in $\beta=30$ successive iterations for PrGA or PtGA, the algorithm is terminated. The run time limit for LINDOGlobal and AlphaECP is set to 3600 seconds (s). Other parameters for AlphaECP and LINDOGlobal are set as default settings.

\subsection{Results and analysis}

As mentioned in the procedure of PTbR, after finding a path, there are three possible ways to send the flow over the generated path, i.e., send possible flow 1) randomly $(\mathbf{R}), 2$ ) one-by-one $(\mathbf{O})$, or 3 ) by a maximum possible amount $(\mathbf{M})$. This creates three different variants of the PTbR-based GA, namely PtGA-R, PtGA-O, and PtGA-M respectively.

To compare the effectiveness of these representation methods, we evaluate these variants, as well as PrGA using a set of 35 MCFP instances. Tables 2-4

present the results of PtGA-R, PtGA-O, PtGA-M, PrGA, LINDOGlobal, and AlphaECP on a total of 35 test problems using cost functions $F_{1}, F_{2}, F_{3}$. The 
Table 2: Results for cost function $F_{1}$.

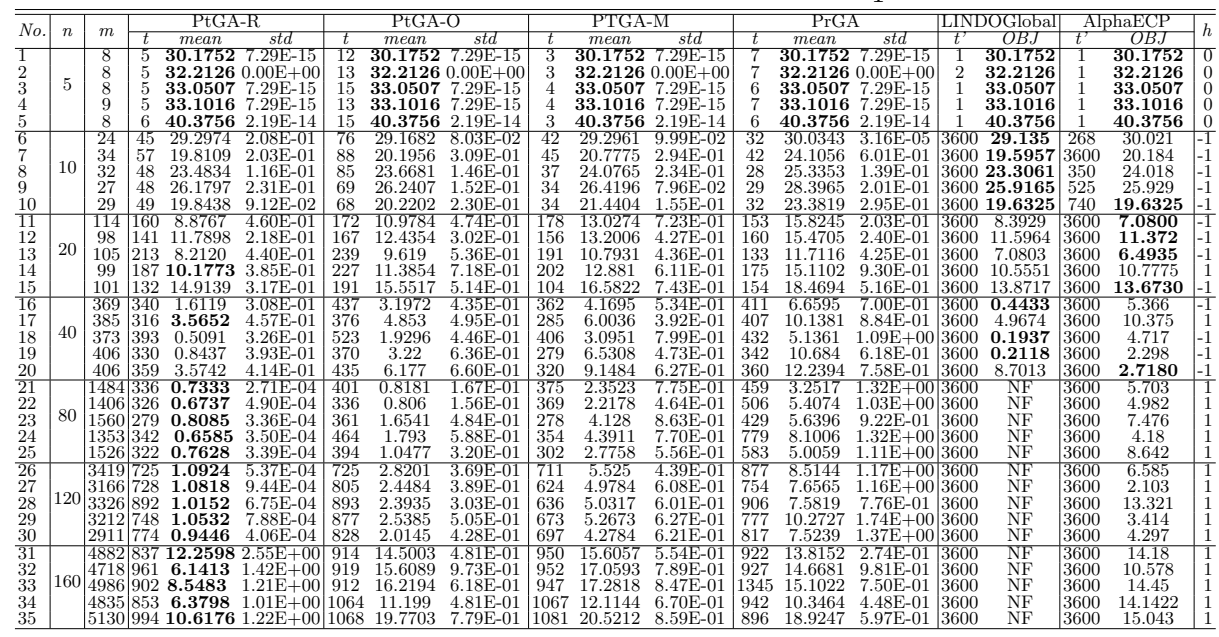

Table 3: Results for cost function $F_{2}$.

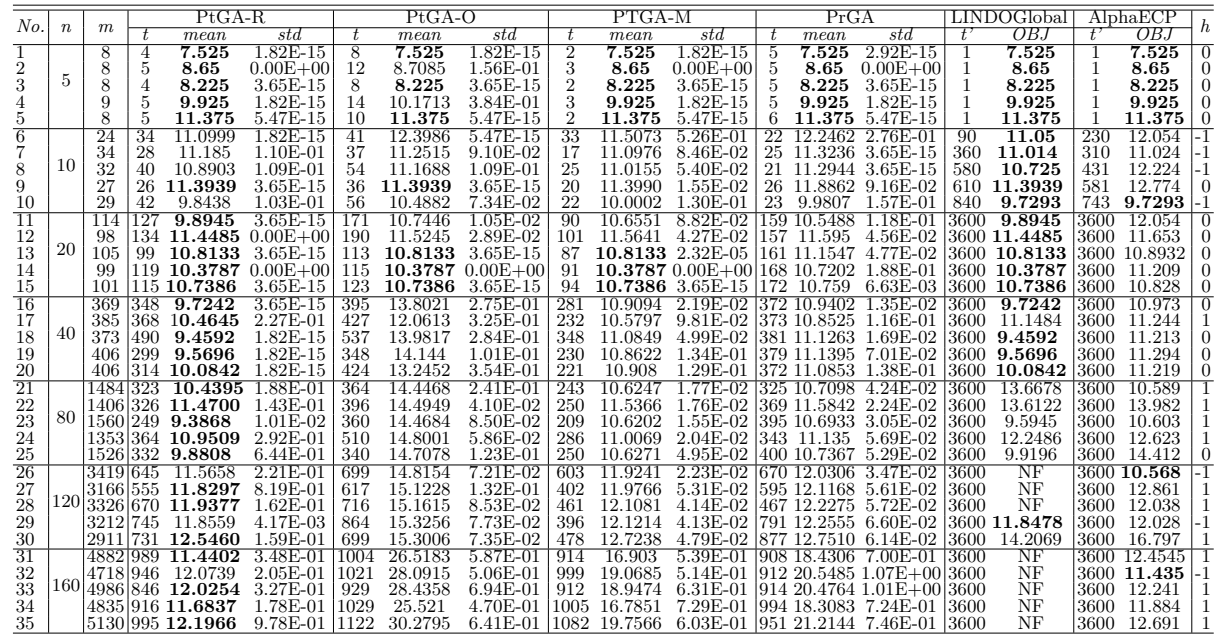

std and $t$ (for PrGA and PtGA) denote the standard deviation of the results and the average of running time in seconds respectively, and the mean represents the average of cost function values over 30 runs. The $t^{\prime}$ and $O B J$ for LINDOGlobal and AlphaECP (exact methods) denote the running time and the cost function value, respectively. "NF" denotes that the mathematical solver cannot find any feasible solution in the time limit of an hour (3600s). The best cost function value for each instance is presented in boldface.

To carry out a comprehensive comparison among PtGA-R, PtGA-O, PtGA$\mathrm{M}$, and PrGA, we use Friedman test [6]. For each function $\left(F_{1}, F_{2}\right.$ and $\left.F_{3}\right)$ we perform the Friedman test with the significance level set to 0.05 , and the results are shown in Table 5. Since the $p$-values in all three functions are almost zero (less than 0.05), there are overall statistically significant differences between the 
Table 4: Results for cost function $F_{3}$.

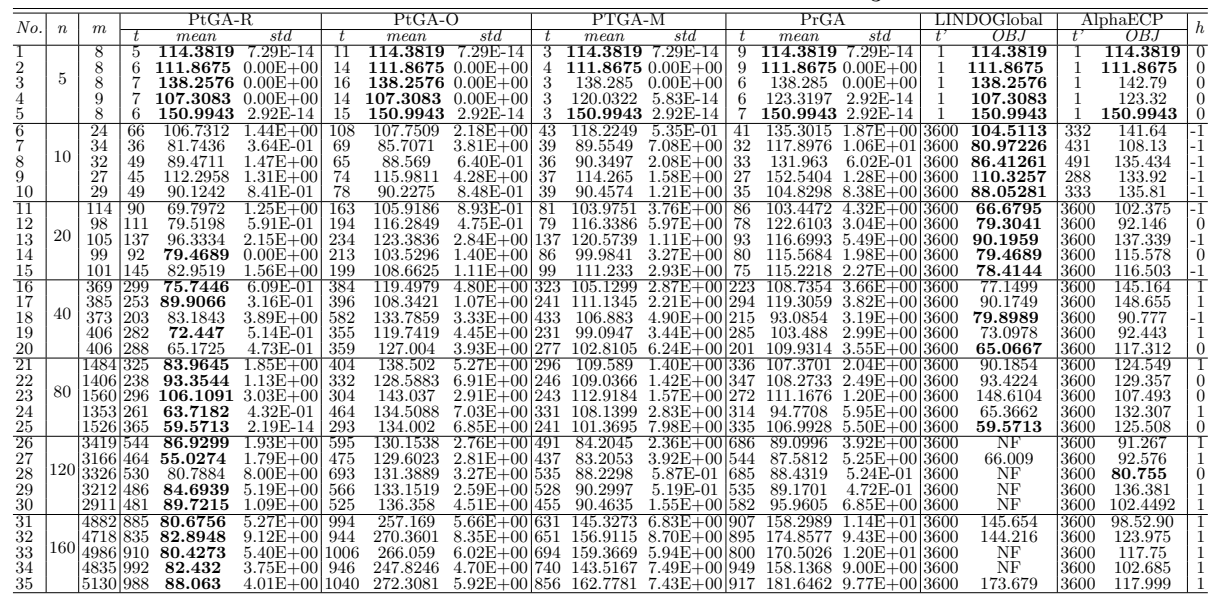

Table 5: The Friedman test's results for PtGA-R, PtGA-O, PtGA-M and PrGA.

\begin{tabular}{c|c|cccc}
\hline \hline & \multirow{2}{*}{$p$-value } & \multicolumn{4}{|c}{ mean column ranks } \\
\cline { 3 - 6 } & & PtGA-R & PtGA-O & PtGA-M & PrGA \\
\hline$F_{1}$ & $0.000 \mathrm{E}+00$ & 16.49 & 34.13 & 50.94 & 60.43 \\
$F_{2}$ & $0.000 \mathrm{E}+00$ & 18.99 & 59.27 & 32.69 & 51.05 \\
$F_{3}$ & $0.000 \mathrm{E}+00$ & 16.28 & 54.94 & 41.05 & 49.72 \\
\hline
\end{tabular}

mean ranks of the algorithms (PtGA-R, PtGA-O, PtGA-M and PrGA). The mean column rank values of the PtGA-R is less than those of the PtGA-O, PtGA-M and PrGA (Table 5) which indicates that PtGA-R's performance is better than those of the other GA variants. It is clearly evident that the superior performance of the PrGA-R comes from utilising PTbR in its procedure and sending a random possible flow.

We also compare the performance of PtGA-R with LINDOGlobal and AlphaECP by applying a one-sample $t$-test with the significance level set to 0.05 . After performing the one-sample $t$-test, if PtGA-R has statistically better or worse performance than that of the mathematical solvers, the parameter $h$ is set to 1 and -1 respectively, otherwise $h$ is set to 0 . The last column of Tables 2-4 presents the value of $h$ for all instances.

For cost function $F_{1}$, Table 2 shows that PtGA-R has better performance on all instances with $n=\{80,120,160\}$ compared with that of PtGA-O, PtGA-M, PrGA, LINDOGlobal and AlphaECP. Furthermore, LINDOGlobal fails to find any feasible solutions when the problem size is increased $(n=\{80,120,160\})$. For $F_{2}$, Table 3 shows that on 28 out of 35 instances (80\%), the PtGA-R has equal or better performance than the two mathematical solvers.

With regard to cost function $F_{3}$, Table 4 shows that even on instances 3 and 4 (small-sized instances), PrGA failed to find the optimal solutions due to the limitations of $\mathrm{PbR}$ in searching the feasible region, which is consistent with our analysis in Subsection 2.1. In all large-sized instances $(n=\{80,120,160\})$, the PtGA-R has similar or better performance than that of the mathematical solvers. 


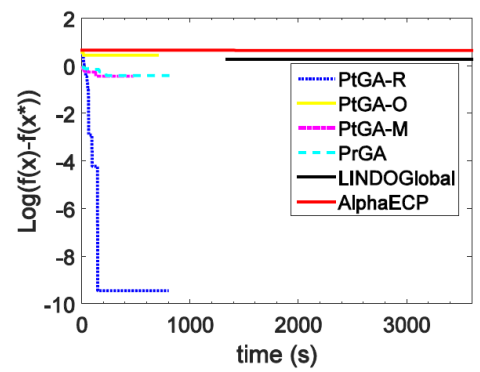

(a) $F_{2}$ on instance No.30.

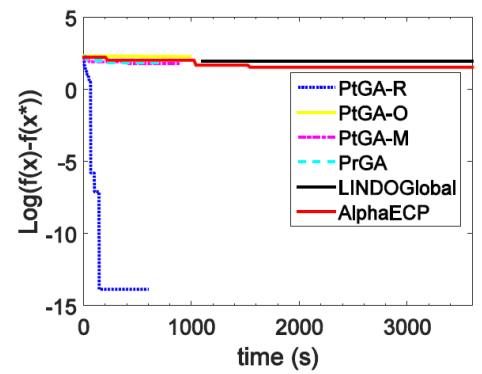

(b) $F_{3}$ on instance No.35.

Fig. 7: Convergence graphs for PtGA, PrGA, LINDOGlobal and AlphaECP.

Fig. 7 shows the convergence graphs of PtGA-R, PtGA-O, PtGA-M, PrGA and the mathematical solvers for large-sized instances on $F_{2}$ and $F_{3}$. Since LINDOGlobal is not able to find any feasible solution for all large-sized problems on $F_{1}$, we are not able to provide the convergence graph for that cost function. As shown in Fig. 7, PtGA-R converges to a good solution faster than other GA variants as well as LINDOGlobal and AlphaECP. Based on Fig. 7, LINDOGlobal cannot find any feasible solution after about 1000s. Once a solution is found, mathematical solvers (specially LINDOGlobal) are not able to improve it.

\section{Conclusion}

This paper has proposed a new encoding scheme called probabilistic tree-based representation (PTbR) for more effective handling of MCFPs. We examine the commonly-used priority-based representation $(\mathrm{PbR})$, and compare it with $\mathrm{PTbR}$ to demonstrate that PTbR is superior to PbR for solving MCFPs. To validate our analysis on these representation schemes, the PTbR-based GA (i.e., PtGA) and PbR-based GA (i.e., PrGA) are evaluated over a set of 35 single-source singlesink network instances with up to five thousand variables. The experimental results demonstrate that PtGA with a random flow (i.e., PtGA-R) has better performance than PrGA on all problem instances. In addition, PtGA-R has also been shown to produce better solutions and have better efficiency than mathematical solvers such as LINDOGlobal and AlphaECP when considering the large-sized instances. For future research, one can focus on solving largesized real-world MCFP using the proposed representation method.

\section{References}

1. Abdelaziz, M.: Distribution network reconfiguration using a genetic algorithm with varying population size. Electric Power Systems Research 142, 9-11 (2017)

2. Ahuja, R.K., Magnanti, T.L., Orlin, J.B.: Network flows: theory, algorithms, and applications. Prentice hall , pp.4-6 (1993) 
3. Aiello, G., La Scalia, G., Enea, M.: A multi objective genetic algorithm for the facility layout problem based upon slicing structure encoding. Expert Systems with Applications 39(12), 10352-10358 (2012)

4. Amiri, A.S., Torabi, S.A., Ghodsi, R.: An iterative approach for a bi-level competitive supply chain network design problem under foresight competition and variable coverage. Transportation Research Part E: Logistics and Transportation Review 109, 99-114 (2018)

5. Burer, S., Letchford, A.N.: Non-convex mixed-integer nonlinear programming: a survey. Surveys in Operations Research and Management Science 17(2), 97-106 (2012)

6. Derrac, J., García, S., Molina, D., Herrera, F.: A practical tutorial on the use of nonparametric statistical tests as a methodology for comparing evolutionary and swarm intelligence algorithms. Swarm and Evolutionary Computation 1(1), 3-18 (2011)

7. Fontes, D.B., Gonçalves, J.F.: Heuristic solutions for general concave minimum cost network flow problems. Networks 50(1), 67-76 (2007)

8. Gen, M., Cheng, R., Lin, L.: Network models and optimization: Multiobjective genetic algorithm approach. Springer Science \& Business Media (2008)

9. Klanšek, U.: Solving the nonlinear discrete transportation problem by minlp optimization. Transport 29(1), 1-11 (2014)

10. Klanšek, U., Pšunder, M.: Solving the nonlinear transportation problem by global optimization. Transport 25(3), 314-324 (2010)

11. Lastusilta, T., et al.: Gams minlp solver comparisons and some improvements to the alphaecp algorithm. Process Design and Systems Engineering Laboratory, Department of Chemical Engineering Division for Natural Sciences and Technology, Abo Akademi University, Abo, Finland (2011)

12. Lin, L., Gen, M.: Bicriteria network design problem using interactive adaptiveweight ga and priority-based encoding method. IEEE Transactions on Evolutionary Computation (2007)

13. Lin, Y., Schrage, L.: The global solver in the lindo api. Optimization Methods \& Software 24(4-5), 657-668 (2009)

14. Lotfi, M., Tavakkoli-Moghaddam, R.: A genetic algorithm using priority-based encoding with new operators for fixed charge transportation problems. Applied Soft Computing 13(5), 2711-2726 (2013)

15. Michalewicz, Z., Vignaux, G.A., Hobbs, M.: A nonstandard genetic algorithm for the nonlinear transportation problem. ORSA Journal on Computing 3(4), 307-316 (1991)

16. Reca, J., Martínez, J., López-Luque, R.: A new efficient bounding strategy applied to the heuristic optimization of the water distribution networks design. In: Congress on Numerical Methods in Engineering CMN (2017)

17. Tari, F.G., Hashemi, Z.: A priority based genetic algorithm for nonlinear transportation costs problems. Computers \& Industrial Engineering 96, 86-95 (2016)

18. Vegh, L.A.: A strongly polynomial algorithm for a class of minimum-cost flow problems with separable convex objectives. SIAM Journal on Computing 45(5), 1729-1761 (2016)

19. Westerlund, T., Pörn, R.: Solving pseudo-convex mixed integer optimization problems by cutting plane techniques. Optimization and Engineering 3(3), 253-280 (2002)

20. Zhang, Y.H., Gong, Y.J., Gu, T.L., Li, Y., Zhang, J.: Flexible genetic algorithm: A simple and generic approach to node placement problems. Applied Soft Computing 52, 457-470 (2017) 